\title{
Relationship between microstructure and mechanical properties of polyether block amide foams
}

\author{
Estève Ernault ${ }^{1}$, Julie Diani ${ }^{1}$, Simon Hallais ${ }^{1}$, Clio \\ Cocquet $^{2}$ \\ ${ }^{1}$ Laboratoire de Mécanique des Solides, CNRS UMR 7649, École Polytechnique, Institut Polytechnique de Paris, \\ Route de \\ Saclay, 91128 Palaiseau, \\ France \\ ${ }^{2}$ Arkema France, CERDATO, Route du Rilsan, 27470 Serquigny, \\ France
}

\author{
Correspondance \\ Estève Ernault \\ Laboratoire de Mécanique des Solides, \\ CNRS UMR 7649, \\ École Polytechnique, Institut Polytechnique de Paris, \\ Route de Saclay, 91128, Palaiseau, France \\ Email: esteve.ernault@polytechnique.edu
}

\section{Funding informations}

Chair "Modeling advanced polymers for innovative material solutions" led by the Ecole polytechnique (l'X) and the Fondation de l'Ecole polytechnique and sponsored by Arkema.

\begin{abstract}
The mechanical behaviors of five polyether block amide foams, obtained by mold-opening foam injection process, were investigated with regard to their microstructures. The materials vary in mass ratios of hard versus soft segments, and/or in process packing time. The resulting microstructures have been characterized in terms of cavity size and shape ratios, by analyzing scanning electron microscope images after careful sample preparation. The foam mechanical responses have been characterized in compression at small and large strain. At small strain, the initial linear part of the stress-strain curve is enhanced firstly by the hard segment mass ratio and secondly by the fineness of the microstructure. Similar results have been obtained at large strain. The foam viscoelasticity at large strain has been characterized by stress relaxation and strain recovery tests, relevant for foam applications. Reduced packing time and pressure have been shown to lead to the presence of undesired large cavities. The morphological defects appear to have a negligible impact on the macroscopic mechanical behavior of the foams at infinitesimal strain, but lead to critical inconsistency at large strain. Furthermore, the mechanical behavior of the tested polyether block amide foams is controlled first by hard vs. soft segments ratio, and second by the microstructure fineness.
\end{abstract}




\section{GRAPHICAL ABSTRACT}

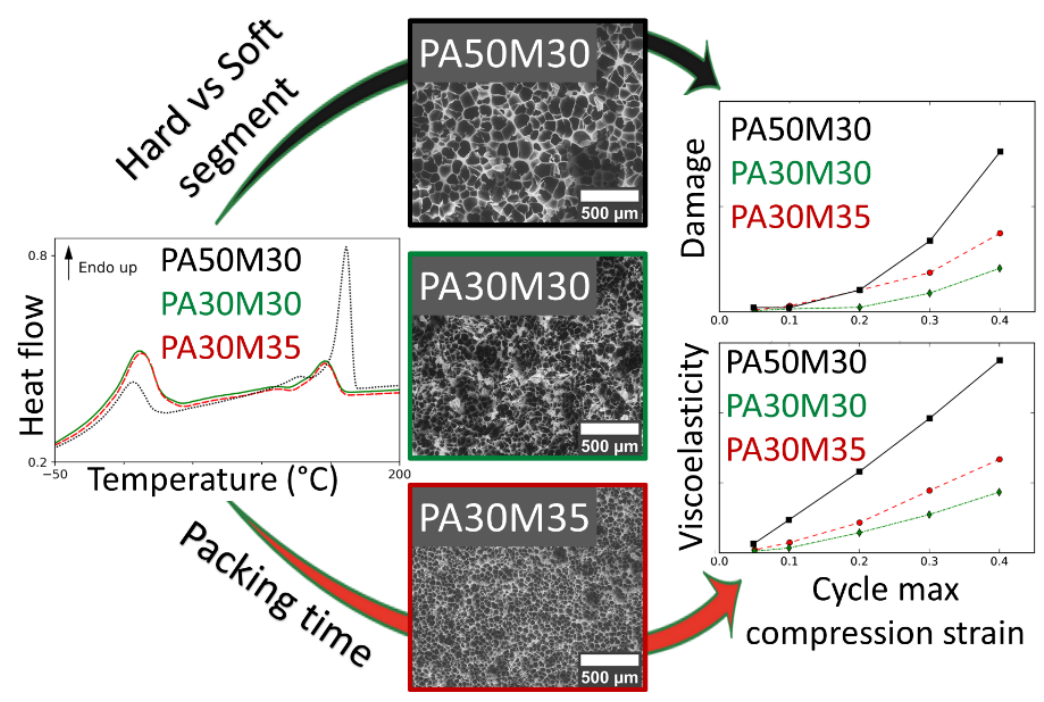

\section{KEWORDS}

foam, microstructure, mechanical behavior, recovery, shape ratio

\section{I | INTRODUCTION}

Cellular materials like wood, cancellous bone, honeycombs... act as useful structures in nature. Cellular polymeric materials have been developed inspired by these natural structures. They are, not only interesting for their weight and cost reductions, but also for their resulting mechanical properties that can be adjusted to targeted applications [1]. Actually, the mechanical behavior of a polymeric foam depends on the mechanical properties of the constitutive phases, and on the parameters characterizing its microstructure like its density [2]. In the case of compression loadings, the cell size and the wall thickness have been shown to be critical parameters $[3,4,5]$.

The cellular microstructure depends on the process. Polymeric foams are obtained using either a chemical or a physical blowing agent. The latter option was chosen in the current study. The foaming mechanism can be described in three steps. First, cell nucleation occurs. Second, the cells grow until all the gas present has diffused into the pores. Last, a step of stabilization is added to maintain the targeted cellular structure $[6,7]$. Several parameters may influence the resulting microstructure. For instance, the presence of crystals in the polymer can act as nucleating agent leading to a heterogeneous cell nucleation, which can increase the cell density $[8,9]$. The nature of the gas, and more specifically its solubility acts on the foam morphology [10].

Actually, cell nucleation and cell growth are competitive phenomena depending on the amount of gas available, the gas concentration affects also the foam structure [4]. Finally, since the polymer viscosity, strength and gas solubility depend on temperature, the latter affects strongly the three steps of the foaming process $[3,11]$.

The PEBAX ${ }^{\circledR}$ foams considered here have been processed by foam injection molding [6] using gas counter pressure and mold opening. The gas counter pressure is used to prevent the nucleation and growth of the cells at the flow front during mold filling. The mold-opening step leads to a pressure drop that triggers the cell nucleation and allows the foam expansion in the direction of the mold opening [12]. The resulting 
Published in Polymer Engineering Science, 61, 1971-1981. https://doi.org/10.1002/pen.25712

microstructures are strongly anisotropic, and the linear compression modulus measured in the expansion direction has been shown to increase with the cell elongation $[13,14]$. The constitutive poly(etherblock- amide) thermoplastic elastomers are made of two oligomers of different physical characteristics link by covalent bond. The soft segments consist in a low glass transition polyether, providing molecular mobility to the copolymer, while the hard polyamide segments act as physical crosslinks and as reinforcing phase [15]. By changing the natures, molecular weights, and mass ratio of hard and soft segments, one may tune the mechanical properties of the copolymer.

A recent study [1] has looked at the impact of gas concentration, injection speed, packing pressure and mold-opening distance, on the compression properties of similar bio-based PEBAX ${ }^{\circledR}$ foams, and has highlighted the role of the microstructure fineness on the material acoustic and mechanical properties. In order to extend these results, it is proposed to look at the effect of the process packing time and of the chemical composition, in terms of soft vs. hard segment ratios, on the resulting microstructures and mechanical properties. Moreover, the mechanical characterization is extended in order to study the stress relaxation and strain recovery at large compressive strain.

In the next section the materials are presented and their microstructures characterized. The chemical and physical microstructures are described by differential scanning calorimetry (DSC). The microstructure morphology is statistically depicted thanks to the analysis of images obtained by scanning electron microscopy (SEM) after careful sample preparation. In section 3, the foams mechanical behaviors are characterized at small and large strain. Several compression tests have been applied including cyclic compressions with increasing maximum strain and recording strain recovery at zero stress at each cycle, and 50\%-compression tests lasting 24 hours followed by strain recovery. The resulting mechanical properties are discussed with regard to the observed microstructures in order to extract the key microstructural features impacting the mechanical behavior of such materials.

\section{II | MATERIALS MICROSTRUCTURE CHARACTERIZATION}

\section{I | Materials presentation}

The polyether block amide (known as trade name PEBAX ${ }^{\circledR}$ ) foams have been supplied by Arkema. They have been processed by mold-opening foam injection molding [1]. Such a process consists in first filling the mold with pressured gas in order to avoid gas nucleation and growth during the injection of the dissolved gas polymer mix. Then, the mold remains closed for a chosen packing time during which the polymer/gas mix flows in the mold and the cell nucleation begins. Finally, the mold is opened enhancing the cell nucleation and cells growth, and leading to the foam expansion [6]. Common process parameters have been chosen with nitrogen as gas blowing agent with a weight percent of $0.6 \%$, a $70^{\circ} \mathrm{C}$ mold temperature, and a mold-opening distance of $12 \mathrm{~mm}$. Materials have been delivered in the form of $15 \mathrm{~mm}$-thick core-skin rectangular plates of dimensions $115 \times 135 \mathrm{~mm}^{2}$.

In an attempt to reduce the packing time, the packing pressure and packing time have been varied. The constitutive hard and soft segments are a polyamide 12 (PA12) and a poly(tetramethylene oxide)(PTMG) respectively. The hard vs. soft segment ratios of three materials have been given by the supplier and are listed in table 1. The melting temperature of commercial references 3533, 4533 and 4033 are 144, 147 and $160{ }^{\circ} \mathrm{C}$ respectively. The material nomenclature writes as PAxMy with $\mathrm{x}$ the $\%$ mass ratio of PA12 and $\mathrm{y}$ the mold packing time in seconds. Note that the impact of the packing time will be assessed by comparison of materials PA30M35, PA30M30 and PA30M25 for their identical packing pressure. 
Published in Polymer Engineering Science, 61, 1971-1981.

https://doi.org/10.1002/pen.25712

\begin{tabular}{cccccc}
\hline & $\begin{array}{c}\text { PEBAX } \\
\text { commercial } \\
\text { reference }\end{array}$ & $\begin{array}{c}\text { PA12 mass } \\
\text { ratio } \\
(\%)\end{array}$ & $\begin{array}{c}\text { PTMG mass } \\
\text { ratio } \\
(\%)\end{array}$ & $\begin{array}{c}\text { Packing } \\
\text { time } \\
(\mathrm{s})\end{array}$ & $\begin{array}{c}\text { Packing } \\
\text { Pressure } \\
\text { (bar) }\end{array}$ \\
\hline PA30M35 & 3533 & 30 & 70 & 35 & 230 \\
PA30M30 & 3533 & 30 & 70 & 30 & 230 \\
PA30M25 & 3533 & 30 & 70 & 25 & 230 \\
PA50M30 & 4033 & 50 & 50 & 30 & 275 \\
PA50M20 & 4533 & 50 & 50 & 20 & 225 \\
\hline
\end{tabular}

TABLE 1 Material compositions and process parameters.

\section{II | DSC Characterization}

To identify the thermal properties of the foams, DSC measurements have been performed with 5 to $10 \mathrm{mg}$ samples encapsulated in aluminum crucibles using the Polyma 214 from Netzsch. All measurements including the baseline with empty crucibles have been performed within a temperature range of $\left[-70^{\circ} \mathrm{C},+200^{\circ} \mathrm{C}\right]$ at a temperature ramp of $10 \mathrm{~K} / \mathrm{min}$. Moreover, two heating ramps have been performed in order to recognize the thermal signature of the molding process.

Samples have been cut at the core of a foam plate excluding the rigid skin. For each foam, at least three samples have been tested and very good reproducibility has been obtained. Therefore, Figure 1 shows only one representative DSC curve of the first and second heating ramp for each material.

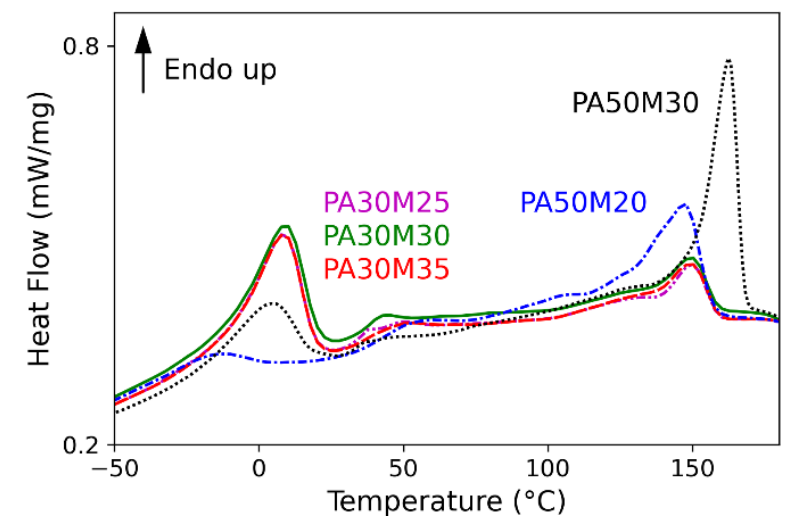

(a)

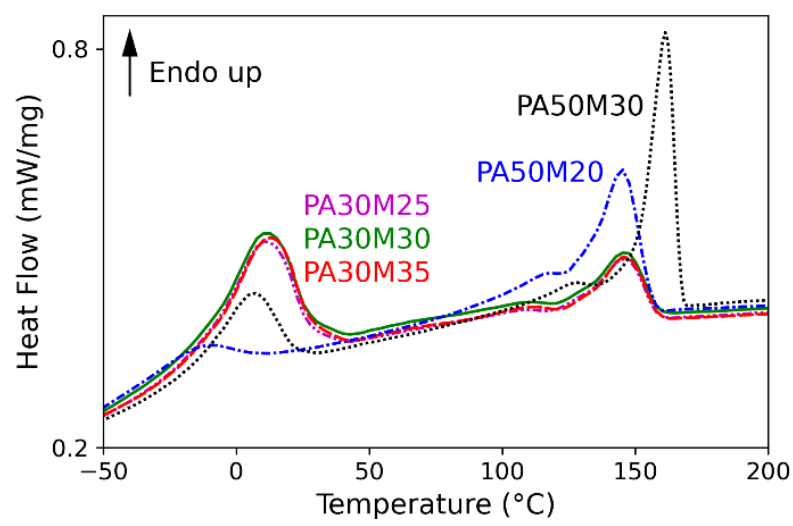

(b)

FIGURE 1 DSC curves of PEBAX ${ }^{\circledR}$ foams performed at $10 \mathrm{~K} / \mathrm{min}$ from $-70^{\circ} \mathrm{C}$ to $200^{\circ} \mathrm{C}$ (a) first heating ramp, (b) second heating ramp. 
Published in Polymer Engineering Science, 61, 1971-1981. https://doi.org/10.1002/pen.25712

For each foam, the second heating ramp displays the molecular mobility and crystallization obtained after common cooling from $200^{\circ} \mathrm{C}$ to $-78^{\circ} \mathrm{C}$ at $10 \mathrm{~K} / \mathrm{min}$, while the differences between the curves of the first and the second heating ramps, point out the impact of the foaming process.

The melting point temperatures of the PTMG and PA12 were extracted at the maximum of the melting peak. The weight percent crystallinity of PA12 has been calculated according to [16]:

$$
X_{c}=\frac{\Delta H_{f}}{\omega_{P A} \Delta H_{f}^{0}}
$$

where $\omega_{\mathrm{PA}}$ is the PA12 mass ratio, $\Delta \mathrm{H}_{\mathrm{f}}$ its melting enthalpy defined by the area of the melting peak and expressed in $\mathrm{J} / \mathrm{g}$ and $\Delta \mathrm{H}_{\mathrm{f}}^{0}=246 \mathrm{~J} / \mathrm{g}$ is the theoretical melting enthalpy of the fully crystallized PA12 [16]. The data extracted from the DSC measures are listed in Table 2.

DSC curves of PA30M25, PA30M30 and PA30M35 superimpose for both the first and second heating ramps, meaning that the packing time is not a parameter affecting the macromolecular microstructure. The melting points of both PTMG and PA12 are lower than the homopolymers ones, due to the presence of an interphase between the soft and hard segments restraining regular crystallization $[17,18,19]$. Considering material PA50M20 and PA50M30, the areas defined by melting peak of PA12 hard segments confirm that these foams contains higher PA12 crystallinity. However, even though the same amount of PA12 has been added for both references, the positions and shapes of their melting peaks differ indicating contrasted crystalline populations. In addition, the melting peak of PTMG is less visible in PA50M20 than for PA50M30. The fact that those differences are observed for both first and second heating ramps imply that they are not related to the process.

\begin{tabular}{|c|c|c|c|c|c|c|}
\hline & \multicolumn{3}{|c|}{ First heating ramp } & \multicolumn{3}{|c|}{ Second heating ramp } \\
\hline & $\begin{array}{c}\text { Melting point } \\
\text { of PTMG } \\
\text { (C) }\end{array}$ & $\begin{array}{c}\text { Melting point } \\
\text { of PA12 } \\
\left({ }^{\circ} \mathrm{C}\right)\end{array}$ & $\begin{array}{c}\text { PA12 } X_{c} \\
(\%)\end{array}$ & $\begin{array}{c}\text { Melting point } \\
\text { of PTMG } \\
\left({ }^{\circ} \mathrm{C}\right)\end{array}$ & $\begin{array}{l}\text { Melting point } \\
\text { of PA12 } \\
\left({ }^{\circ} \mathrm{C}\right)\end{array}$ & $\begin{array}{c}\text { PA12 } X_{c} \\
(\%)\end{array}$ \\
\hline PA30M35 & $9.4 \pm 0.6$ & $148.9 \pm 0.2$ & $14.6 \pm 2$ & $11.9 \pm 0.5$ & $147.8 \pm 1$ & $14.2 \pm 1$ \\
\hline PA30M30 & $10.2 \pm 0.6$ & $149.6 \pm 0.5$ & $15.5 \pm 0.5$ & $11.7 \pm 0.6$ & $148 \pm 0.9$ & $14.8 \pm 1$ \\
\hline PA30M25 & $9 \pm 0.5$ & $149.8 \pm 2$ & $12.3 \pm 3$ & $10.1 \pm 0.9$ & $147.5 \pm 0.6$ & $14.7 \pm 0.6$ \\
\hline PA50M30 & $4.5 \pm 0.3$ & $151.3 \pm 0.1$ & $21.3 \pm 0.6$ & $7.1 \pm 0.4$ & $161.4 \pm 0.2$ & $21.5 \pm 0.5$ \\
\hline PA50M20 & $-12.2 \pm 0.8$ & $146.3 \pm 1$ & $21.3 \pm 4$ & $-9.9 \pm 0.9$ & $144.8 \pm 0.6$ & $25.6 \pm 2$ \\
\hline
\end{tabular}

TABLE 2 Crystalline phase melting point temperatures and material crystallinity extracted from DSC curves shown in Figure 1.

The interphase between soft and hard segments depends on the crystalline phases of PTMG and PA12, and lowering the molecular weight of PTMG may soften the separation between the hard and soft phases 
Published in Polymer Engineering Science, 61, 1971-1981.

https://doi.org/10.1002/pen.25712

[20]. This would explain why the melting peak of PA12 in PA50M20 is lower and wider than in PA50M30. Actually, softening the hard and soft phases separation may reduce crystal perfection and increase its polydispersity [21].

\section{III | Foam morphology Characterization}

The morphologies of the foams have been described thanks to images obtained with a Field Emission Gun Environmental Scanning Electron Microscope (FEG-ESEM) FEI Quanta 650. SEM has been preferred to optical microscopy for its greater field depth. The samples have been carefully prepared to avoid surface damage. Cylinders of diameter and height of $15 \mathrm{~mm}$ have been immersed in liquid nitrogen for 30 minutes before being fractured in order to obtain clean surfaces. Note that SEM samples and compression test specimens have been cut in the same area, avoiding the edges of the plates. The fractured surfaces have been covered with a $20 \mathrm{~nm}$ gold layer in order to avoid charge accumulation effects in SEM. Images have been performed at low vacuum $(100 \mathrm{~Pa})$, with an accelerating voltage of $15 \mathrm{keV}$, a probe current of $0.26 \mathrm{nA}$, a field width of $500 \mu \mathrm{m}$, and a pixel dwell time of $5 \mu \mathrm{s} /$ pixel. The image definition is $4092 \times 3516$ pixels. To capture a large enough area and better characterize the morphology some stitching with $10 \%$ overlapping were also performed.

Figure 2 shows representative images of the pores of the foam when cut perpendicular to the direction of mold-opening and parallel to the melt flow direction, while Figure 3 presents images of the microstructure of the foam cut along the mold-opening direction. First, material PA50M20 shows very large defects (Figure 3). These macro-pores have been observed spread unevenly across the microstructures and are also present, in a lower extent, in material PA30M25, and shows that a minimum packing time is necessary in order to obtain regular homogeneous microstructures. Note that the pressure may also have an impact on the cell growth rate $[22,4]$, which may explain the significantly larger defects observed in material PA50M20. Second, the foams appear as strongly anisotropic with elongated cells parallel to the mold-opening direction. In order to quantify the geometry of the cells, two quantities are defined. Assuming that cells may be represented by an average oblong spheroid characterized by a short diameter $2 \times \bar{a}$ and a long diameter $2 \times \bar{b}$, the length $\bar{a}$ may be calculated as follow:

$$
\bar{a}=\sqrt{\frac{3 \bar{A}}{2 \pi}}
$$

with $\bar{A}$ the average pores area when the microstructure is cut parallel to the flow direction, and the length

$\bar{b}$ measured on the images of samples cut in the direction of mold-opening. The cavity elongation is then characterized by a mere shape ratio $\rho=\bar{b} / \bar{a}$. For each material, the average area, $\bar{A}$, has been calculated over at least 174 cells identified and characterized by image analysis, using ImageJ software [23] on stitched images as Figure 4 illustrates. 
Published in Polymer Engineering Science, 61, 1971-1981.

https://doi.org/10.1002/pen.25712

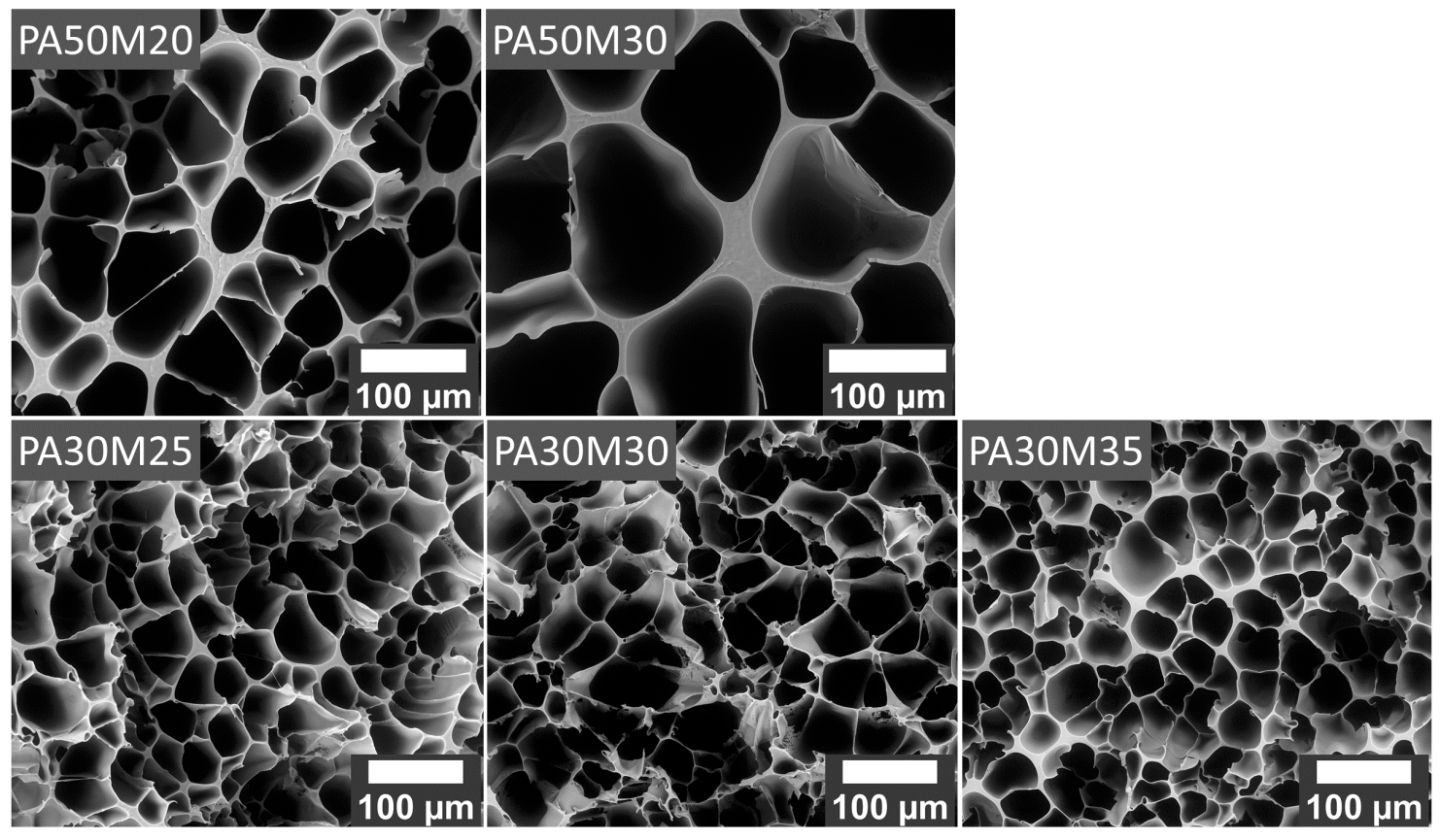

FIGURE 2 SEM backscattered electron image of PEBAX ${ }^{\circledR}$ foams cut perpendicular to the direction of mold-opening.

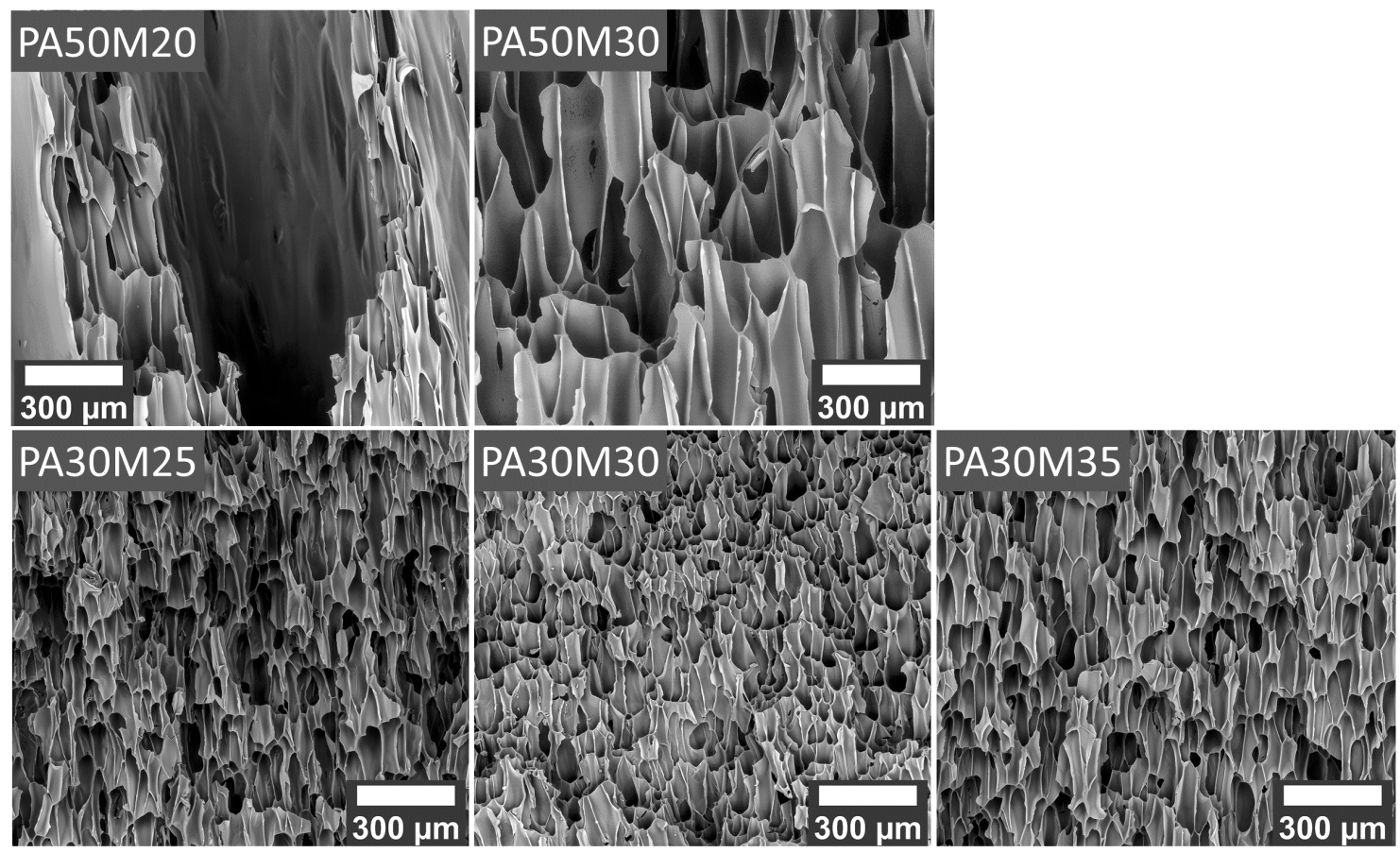

FIGURE 3 Stitching of 3 x 3 SEM backscattered electron image of PEBAX ${ }^{\circledR}$ foams cut along the moldopening direction.

The lengths $\bar{b}$ have been obtained as the averaged length of at least 20 cells. To ensure the 
Published in Polymer Engineering Science, 61, 1971-1981.

https://doi.org/10.1002/pen.25712

representativeness of the morphological parameters, only cells fractured parallel to the observed surface are considered, and the macropores discussed earlier were not considered. The quantitative values characterizing the morphologies are listed in Table 3.

\begin{tabular}{cccc}
\hline & $\begin{array}{c}\text { Oblong pore short radius } \\
\bar{a}(\mu \mathrm{m})\end{array}$ & $\begin{array}{c}\text { Oblong pore long radius } \bar{b} \\
(\mu \mathrm{m})\end{array}$ & $\begin{array}{c}\text { Shape ratio } \\
\rho=\bar{b} / \bar{a}\end{array}$ \\
\hline PA30M35 & $17 \pm 6$ & $99 \pm 33$ & 5.8 \\
PA30M30 & $20 \pm 10$ & $101 \pm 19$ & 5.1 \\
PA30M25 & $19 \pm 6$ & $102 \pm 26$ & 5.4 \\
PA50M30 & $54 \pm 16$ & $283 \pm 57$ & 5.2 \\
PA50M20 & $29 \pm 9$ & $165 \pm 51$ & 5.7 \\
& & & \\
\hline
\end{tabular}

TABLE 3 Average dimensions of the oblong shape pores characterizing the foam morphologies.

The comparison of the foam morphologies of PA30M30 and PA50M30 having the same packing time allows us to study the influence of the quantity of PA12 on cell morphology. Although PA50M30 has been processed at a higher packing pressure, its average cell size is significantly larger than PA30M30 (Table 3), which is likely due to the amount of PA12 acting as a counter effect [1]. Since the morphology of the crystalline phase of the poly(ether-block-amide) can influence nucleation [11], a significant impact of the ratio of hard vs. soft segments on the foam morphology was expected.

The difference of the cell size between PA50M20 and PA50M30 may be explained by the difference of the hard segment arrangements. Although both materials PA50M30 and PA50M20 contain 50 weight $\%$ of PA12, Figure 1 shows that their physical arrangement are different. Note that despite the contrasted cavity sizes reported in Table 3, their aspect ratios are of the same order, with PA30M35 and PA50M20 showing cavities slightly more oblong.

Finally, another effect of the packing time can be observed when comparing the morphologies of PA30M30 and PA30M35 in Figure 4, which represents a distant larger view in the direction perpendicular to the mold-opening. Increasing the packing time seems to improve the foam homogeneity. This was observed also when preparing the samples, as it was easier to obtained a cleavage plane by fragile fracture for material PA30M35 than for material PA30M30 (Figure 4). 

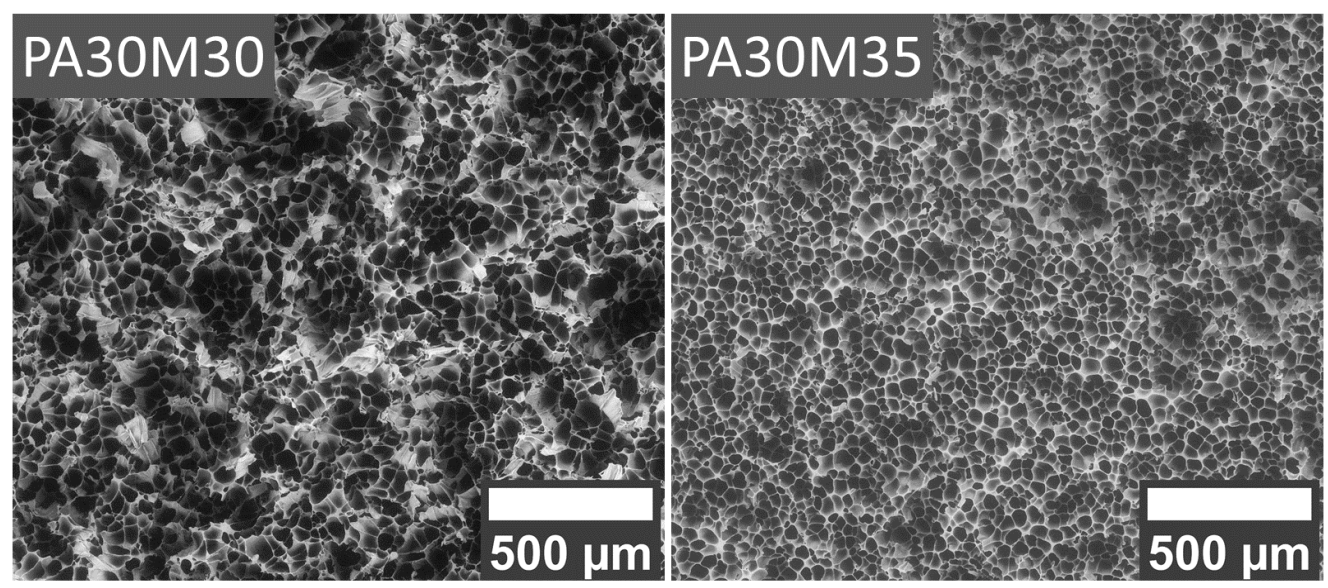

FIGURE 4 Stitching of 4 x 4 SEM backscattered electron image of PEBAX ${ }^{\circledR}$ foams cut perpendicular to the direction of mold-opening.

\section{III | MECHANICAL BEHAVIOUR}

\section{I | Mechanical tests}

Compression mechanical tests were carried out with an Instron 5967 tensile machine on cylinder specimens of $21.5 \mathrm{~mm}$ diameter and $15 \mathrm{~mm}$ height. Sample skin layers of $0.8 \mathrm{~mm}$ have been kept in order to ensure that sample opposite sides are parallel. The dimensions of the sample were chosen large enough to be representative of the observed cellular microstructure. Compression stress has been applied in moldopening direction only.

In order to characterize the material linear stress-strain responses, the specimens were submitted to compressions at a low constant crosshead speed of $0.1 \mathrm{~mm} / \mathrm{min}$ in order to limit the viscoelasticity. Three samples were tested three times. For each test, the Young modulus has been calculated by linear regression of the stress-strain response for the strain ranging between 0.005 and 0.01 .

At large strain, specimens of the same dimensions were submitted to cyclic uniaxial compressions at a constant crosshead speed of $1 \mathrm{~mm} / \mathrm{min}$ with the maximum strain increasing of $10 \%$ at each cycle. In order to estimate the residual strain recovery after each cycle, a strain recovery step at zero stress was included after each unloading step. The durations of the strain recovery steps are listed in Table 4. Finally, samples were also submitted to a $50 \%$ compression for 24 hours during which the stress relaxation was recorded. After the 24-hour load, the samples were unloaded and stress-free strain recoveries have been monitored by filming the specimens with a Spark Series SP-20000M 20 MPx camera equipped with a telecentric lens, and post-processing the $4192 \times 3714$ pixels images with Matlab [24]. 
Published in Polymer Engineering Science, 61, 1971-1981.

https://doi.org/10.1002/pen.25712

\begin{tabular}{cc}
\hline $\begin{array}{c}\text { Maximum applied strain } \\
(\%)\end{array}$ & $\begin{array}{c}\text { Strain recovery time } \\
(\mathrm{min})\end{array}$ \\
\hline 5 & 120 \\
10 & 180 \\
20 & 240 \\
30 & 300 \\
40 & 360 \\
50 & 480 \\
\hline
\end{tabular}

TABLE 4 Durations of the strain recovery steps at zero stress when applying large deformation cyclic compression tests.

\section{II | Linear elasticity}

The average Young modulus and its standard deviation are shown in Figure 5. As one could expect, materials with $50 \%$ mass ratio of PA 12 hard segments are significantly stiffer than materials with $30 \%$ only. Actually, the stress measured during the linear elastic part of the material stress-strain response, results from the bending of the cell walls $[2,25]$, which explains why the ratio PA12/PTMG has a significant impact on the elastic modulus. More interestingly, when comparing materials PA50M20 and PA50M30, one reads that the large cavities randomly spaced out in PA50M20 have little impact on the Young modulus. While, material PA50M20 shows much smaller cavities than material PA50M30, its Young modulus is not that significantly higher. Therefore, the stiffer Young modulus shown by material PA50M20 is most likely due to the more elongated pores characterized by the shape ratio of 5.7 compared to 5.2 for material PA50M30. Same results have been obtained for materials with $30 \%$ of hard segments. Materials PA30M25 and PA30M30 that displayed similar cavities sizes and aspect ratios present similar Young moduli despite the fact that material PA30M25 microstructure revealed some defects like material PA50M20. Moreover, the more oblong cavities present in material PA30M35 are likely the cause of the stiffer response. Since cavities displayed by PA50Mx materials are significantly larger, one notes that the amount of rigid segments has a first order impact on the Young modulus, while the microstructure morphology counts as a second order one. 


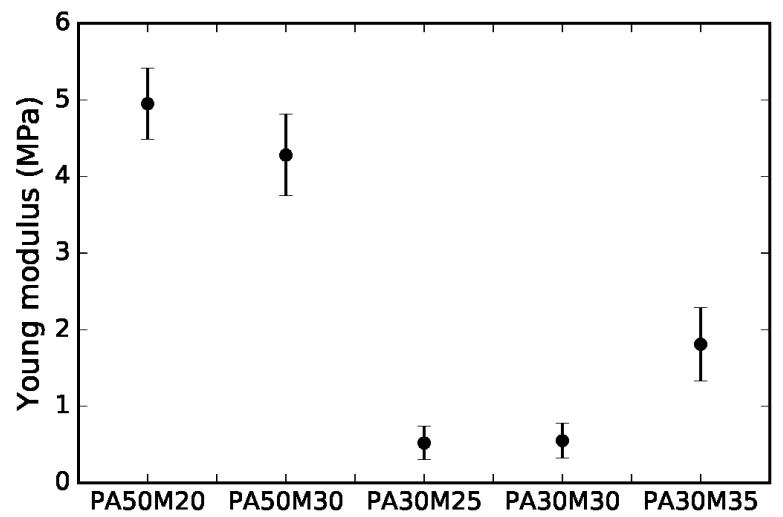

FIGURE 5 Average young modulus characterized over five samples submitted to uniaxial compressions, the error bars indicate the standard deviation.

\section{III | Large deformation non linear behavior}

Foams were submitted to cyclic uniaxial compression tests at large strain. At each cycle, the maximum compression was increased and a stress-free relaxation step was set to monitor the residual strain recovery. An illustration of the difference of reproducibility between material PA30M25 presenting large defects and material PA50M30, is shown in Figure 6. It was noticed that the large defects witnessed in PA30M25 and PA50M20 induce poor experimental reproducibility at large strain. Consequently, only materials PA50M30, PA30M30 and PA30M35, will be studied in what follows.

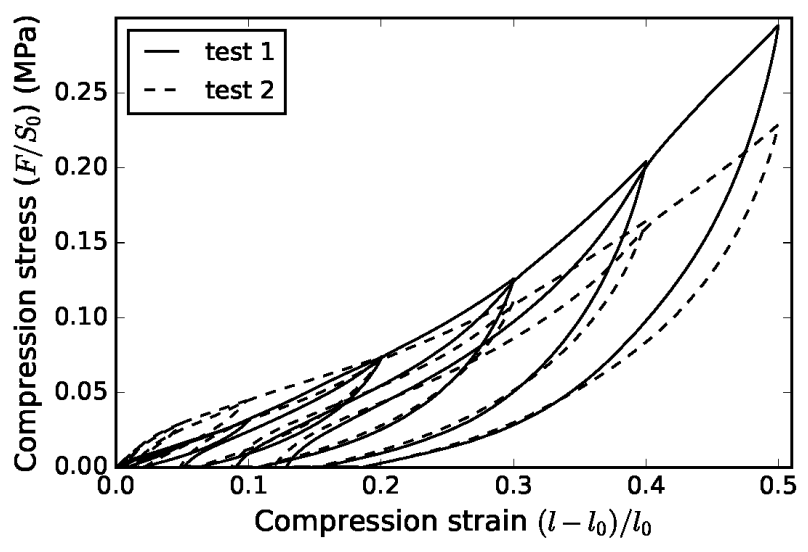

(a)

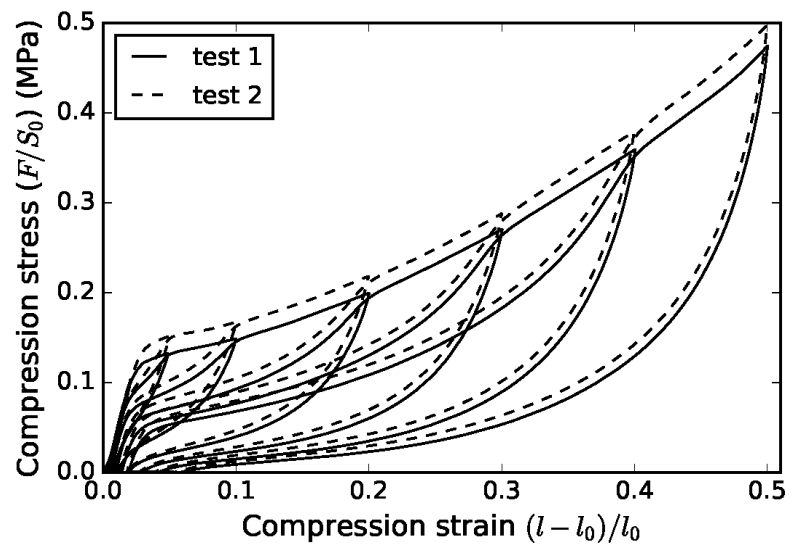

(b)

FIGURE 6 Comparison of the experimental reproducibility obtained for cyclic uniaxial compression stressstrain responses of (a) material PA30M25 and (b) material PA50M30.

Figure 7 shows the cyclic stress-strain responses in compression of materials PA50M30, PA30M30 and PA30M35. The loading compression curves are typical of mechanical responses of polymeric foam materials under compression. It can be described into several parts. The first one is the linear elastic behavior which has been discussed in the previous paragraph (section 3.2). The second part of loading curve, observed as a plateau, characterizes the nonlinear behavior caused by cell wall buckling. As the compression load 
Published in Polymer Engineering Science, 61, 1971-1981.

https://doi.org/10.1002/pen.25712

increases, foam densification due to the instable collapse of the walls occurs until the foam is fully dense $[2,25,26]$. In agreement with results obtained at small strain, PA50M30 is significantly stiffer than the two other materials for the larger ratio of hard segments, and PA30M35 is stiffer (higher elastic modulus and higher compression strength) than PA30M30 due to difference of foam morphology.

Looking at the overall loading-unloading curves, one notes that when first submitted to a level of strain never applied, the foams undergo some significant softening. The damage is reactivated only when exceeding the maximum strain already applied. Once the damage softening evacuated, the materials show viscoelastic behaviors with different loading and unloading responses that reproduce well when more cycles are considered [1]. In PEBAX ${ }^{\circledR}$ foams these features are associated to permanent yielding and fracture of the cell walls buckling under compression [25].

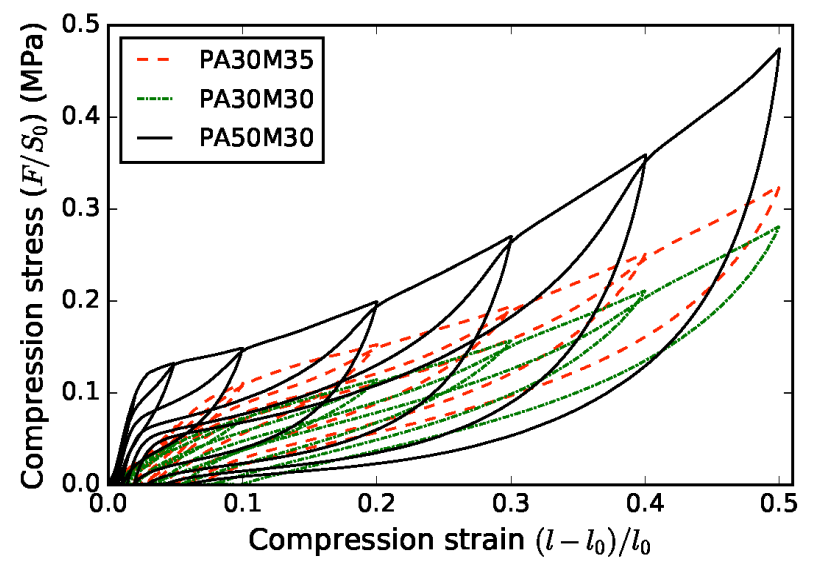

FIGURE 7 Cyclic uniaxial compression stress-strain responses.

For each material, and each cyclic maximum strain, one may estimate the hysteresis area resulting from material damage and the hysteresis area due to the material viscoelasticity. A scheme depicting how these areas are calculated is displayed in Figure 8 for the $40 \%$ strain cycle.

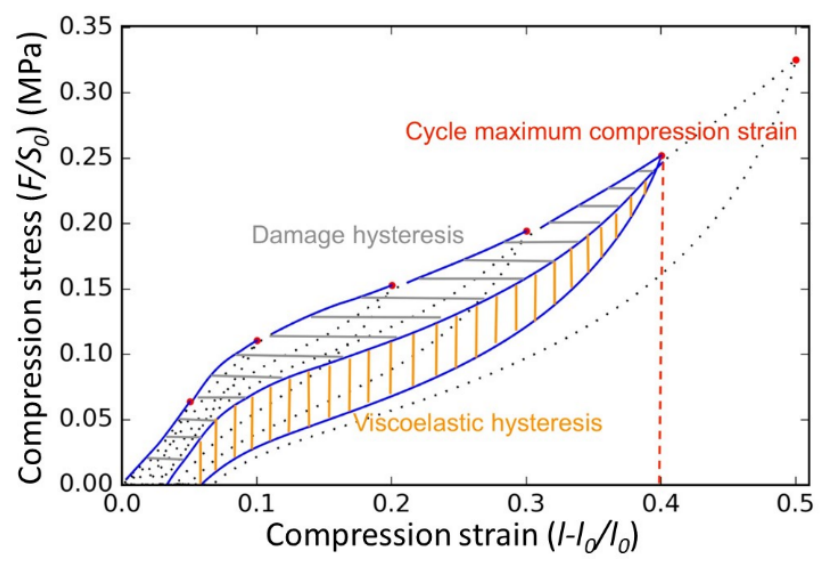

FIGURE 8 Scheme of the damage hysteresis and the viscoelasticity hysteresis that are calculated from data plotted in Figure 7.

Figure 9 presents the damage and viscoelasticity hystereses. They both have the same trends increasing with the maximum strain applied and with the material stiffness. The damage hystereses area present the 
Published in Polymer Engineering Science, 61, 1971-1981.

https://doi.org/10.1002/pen.25712

cumulative damage associated to permanent microstructural changes. Materials PA30M30 and PA30M35 having common chemical compositions, show similar shape curves, and the higher level of damage witnessed in material PA30M35 is probably linked to its higher strength resulting from the cellular microstructure.

In order to better evaluate the material damage and viscoelasticity, the recovery of the residual strain at zero stress at the end of each cycle has been monitored (Figure 10). First, the instantaneous residual strain after each compression cycle is larger for foam PA30M30 than for the two other ones. Actually, the more compliant behavior of the former foam is probably favoring the buckling of the cell walls enhancing the residual strain $[5,27]$. Second, the strain recovery kinetics characterizing the material viscoelasticity of the foams show that recovery is faster and more complete for material PA50M30 which is due to a larger elasticity/viscoelasticity stiffness ratio. The larger amount of hard segments has provided with a significantly stiffer material with enhanced elasticity that favors the foam recovery. However, the recovery remains incomplete even at relatively small deformation $5 \%$, which is the sign of some permanent damage in the material. Some authors $[2,27]$ have also indicated the presence of remaining collapsed zones after compression tests. Similarly, to the linear elasticity, the residual strain recovery is improved first by increasing the amount of hard segments and second by the refinement of the microstructure.

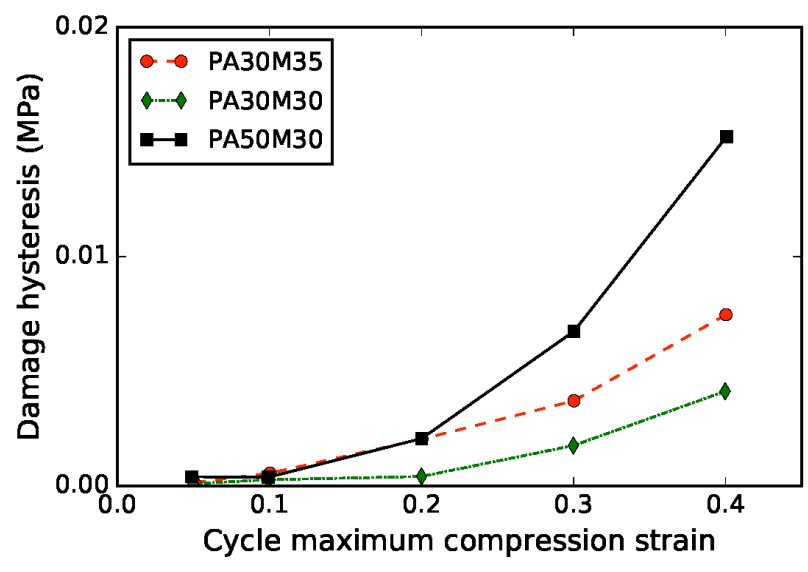

(a)

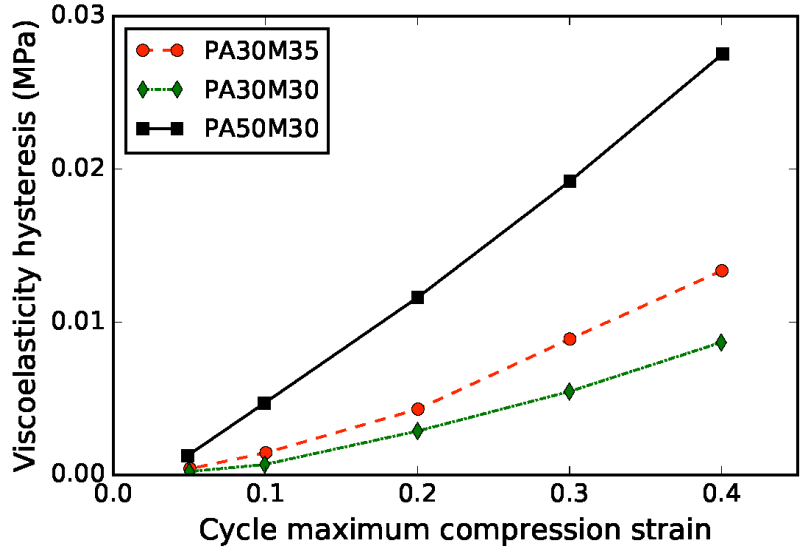

(b)

FIGURE 9 Damage hysteresis and viscoelasticity hysteresis calculated according to Figure 8 for cyclic stress-strain responses displayed in Figure 7.

\section{IV | Long compression stress relaxation and strain recovery}

As a last mechanical test, the material strain recovery has been recorded during 24 hours after applying a 50\% compression strain during 24 hours and releasing the stress. During the applied loading, it was possible to record the stress relaxation as well (Figure 11).

The stress relaxation kinetics are dependent on both the amount of hard segments and of the cells characteristics. For the foam PA50M30 presenting the larger amount of hard segments, the stress relaxation is faster, which is probably due to the different viscoelastic properties of PA12 vs. PTMG. The impact of the cellular morphology on the molecular mobility may be seen when comparing materials PA30M30 and PA30M35. The amount of stress relaxation seems larger for PA30M35 than for PA30M30, probably due to its enhanced stiffness. The molecular mobility appears as significantly slowed down for material PA30M35, since the equilibrium plateau had not been reached within the 24 hours allocated.

Finally, the post-loading strain recovery is shown in Figure 12. The first part of the recovery is guided 
by the compression platen, still in contact with the sample, which is slowly lifted at the constant crosshead speed of $1 \mathrm{~mm} / \mathrm{min}$. During this part of the test, the material strain recovery is mostly elastic which explains that its kinetic is the same as the displacement of the compression platen for every material. The points at which the experimental curves change from concave to convex shapes designate the loss of contact between the compression platen and the specimen. At that point the elastic strain is entirely recovered and as one could expect this points order is driven by the different stiffnesses of the materials. Past that point, material PA50M30 displays the faster and more complete recovery. Then, material PA30M30 shows the second faster recovery, while material PA30M35 is still recovering very slowly after 24 hours. These results are in agreement with the previous stress-free strain recoveries presented in Figure 10.

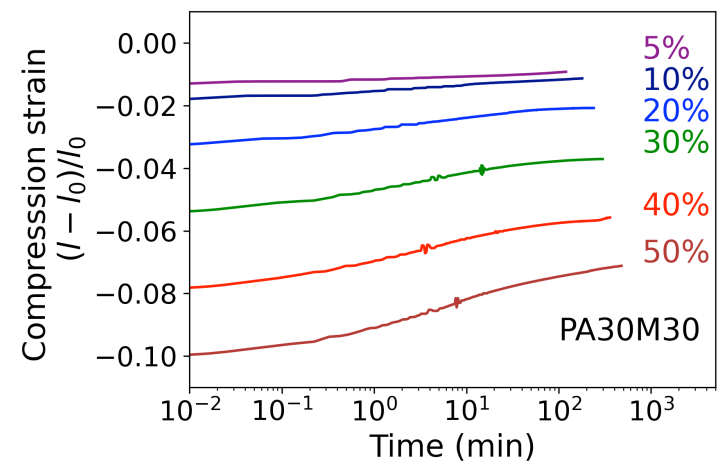

(a)

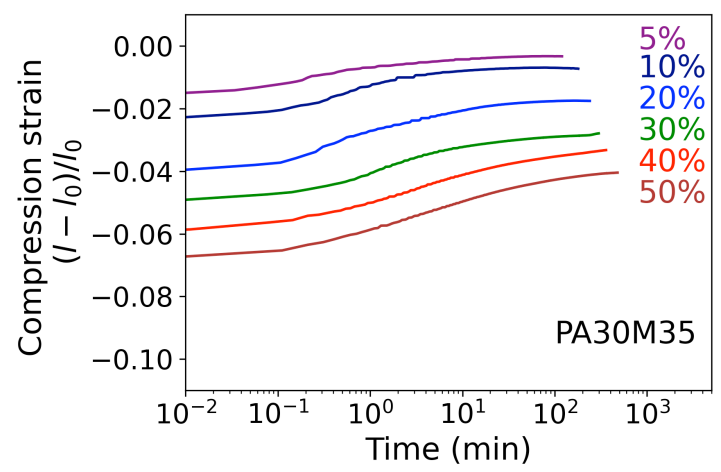

(b)

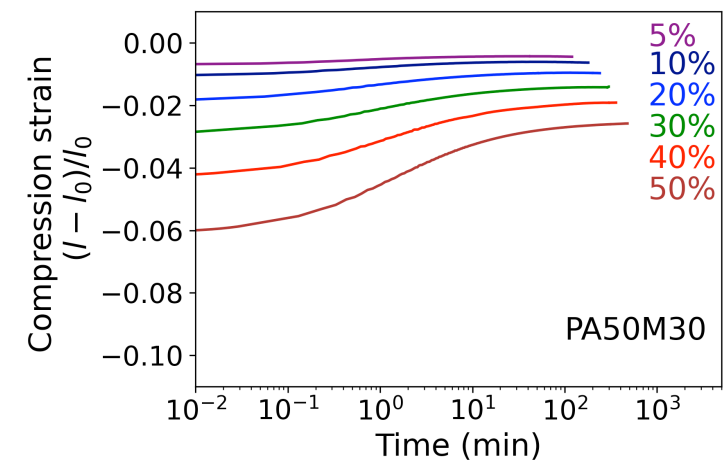

(c)

FIGURE 10 Strain recovery at zero stress after each cycle for tests displayed in Figure 7. Figure has been modified according to reviewer 1 and 2 comments. 


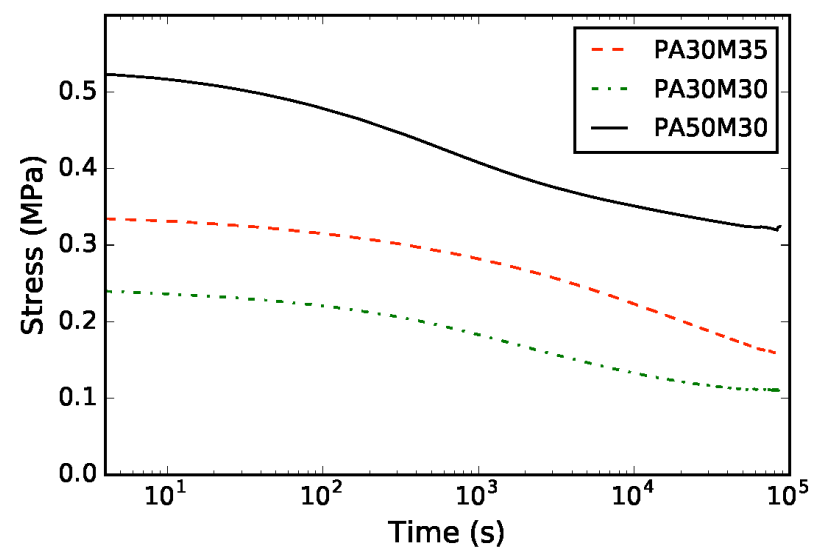

FIGURE 11 Stress relaxation vs. time for samples submitted to uniaxial compressions at 50\%.

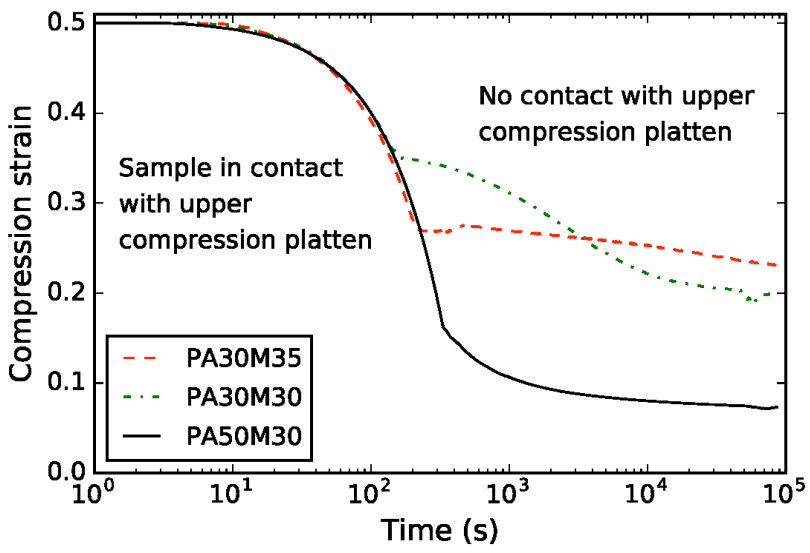

FIGURE 12 Strain recovery recorded with respect to time when unloading and further zero-stress step for samples previously submitted to 50\% uniaxial compressions during 24 hours. Figure has been modified according to reviewer 1 comments.

\section{IV | CONCLUSION}

The influence of the packing time and of the hard (PA12) with respect to soft (PTMG) segment ratios, on the microstructure and the resulting mechanical properties of five PEBAX ${ }^{\circledR}$ processed by foam injection molding, have been investigated. The DSC analyses of the physical macromolecular microstructures of the foams have shown a negligible effect of the process packing time but a significant impact of the amount of hard vs. soft segments. The cellular morphology characterized by scanning electron microscopy highlighted that longer packing time leads to more homogeneous cell organization, whereas increasing hard segment content drives to larger cells due to the enhanced polymer melt strength.

Compression tests at low strain showed that higher hard segment content leads to higher elastic modulus increasing the cell walls resistance to bending. Cyclic compression at large strain allowed us to study the nonlinear responses of the materials resulting from cell wall buckling creating collapsed zones driving ultimately to foam densification. The resulting damage was characterized in terms of damage hysteresis and 
permanent strain, while the material viscoelasticity at large strain was monitored by stress-free strain recovery and constant strain stress recovery. The comparison of the mechanical responses and foam morphology lead to a first order impact of the chemical composition of the Poly(ether-block-amide), the amount of rigid segments being a particularly important parameter. The cell geometrical characteristics appear as a second order impact still meaningful when the same chemical compositions are considered.

\section{ACKNOWLEDGMENT}

This action benefited from the support of the Chair "Modeling advanced polymers for innovative material solutions" led by the Ecole polytechnique (l'X) and the Fondation de l'Ecole Polytechnique and sponsored by Arkema.

\section{CONFLICT OF INTEREST}

The author declares that there is no conflict of interest that could be perceived as prejudicing the impartiality of the research reported.

\section{DATA AVAILABILITY STATEMENT}

The raw data required to reproduce these findings are available to download from Ernault, Esteve (2020), "raw data Relationship between microstructure and mechanical properties of PEBAX foams", Mendeley Data, V1, doi: 10.17632/7y552wrmxp.1.

\section{REFERENCES}

[1] G. Wang, G. Zhao, G. Dong, Y. Mu, C. B. Park, G. Wang, Eur. Polym. J. 2018, 103, 68-79.

[2] I. J. Gibson, M. F. Ashby, Proc. R. Soc. Lond. Math. Phys. Sci. 1982, 382, 43-59.

[3] J. Zhao, Q. Zhao, L. Wang, C. Wang, B. Guo, C. B. Park, G. Wang, Eur. Polym. J. 2018, 98, 1-10.

[4] G. Wang, J. Zhao, G. Wang, L. H. Mark, C. B. Park, G. Zhao, Eur. Polym. J. 2017, 95, 382-393.

[5] Y. Chen, R. Das, M. Battley, Int. J. Eng. Sci. 2017, 120, 220-240.

[6] M. Rohleder, F. Jakob, in Spec. Inject. Molding Tech., Elsevier, 2016, pp. 53-106.

[7] M. Li, J. Qiu, H. Xing, D. Fan, S. Wang, S. Li, Z. Jiang, T. Tang, Polymer 2018, 155, 116-128.

[8] V. Shaayegan, G. Wang, C. B. Park, Chem. Eng. Sci. 2016, 155, 27-37.

[9] A. Wong, Y. Guo, C. B. Park, J. Supercrit. Fluids 2013, 79, 142-151.

[10]T. Ishikawa, K. Taki, M. Ohshima, Polym. Eng. Sci. 2012, 52, 875-883.

[11] M. R. Barzegari, N. Hossieny, D. Jahani, C. B. Park, Polymer 2017, 114, 15-27.

[12] J. W. S. Lee, R. E. Lee, J. Wang, P. U. Jung, C. B. Park, Chem. Eng. Sci. 2017, 167, 105-119.

[13] V. Bernardo, E. Laguna-Gutierrez, A. Lopez-Gil, M. A. Rodriguez-Perez, Mater. Des. 2017, 114, 8391.

[14] A. T. Huber, L. J. Gibson, J. Mater. Sci. 1988, 23, 3031-3040.

[15] D. J. Buckwalter, J. M. Dennis, T. E. Long, Prog. Polym. Sci. 2015, 45, 1-22.

[16] V. I. Bondar, B. D. Freeman, J. Polym. Sci. Part B Polym. Phys. 1999, 37, 2463-2475.

[17] T. Ishikawa, S. Nagai, N. Kasai, J. Polym. Sci. Polym. Phys. Ed. 1980, 18, 1413-1419. 
Published in Polymer Engineering Science, 61, 1971-1981.

https://doi.org/10.1002/pen.25712

[18] G. R. Hatfield, Y. Guo, W. E. Killinger, R. A. Andrejak, P. M. Roubicek, Macromolecules 1993, 26, 6350-6353.

[19] J. P. Sheth, J. Xu, G. L. Wilkes, Polymer 2003, 44, 743-756.

[20]A. M. Castagna, A. Pangon, T. Choi, G. P. Dillon, J. Runt, Macromolecules 2012, 45, 8438-8444.

[21] M. Xie, Y. Camberlin, Makromol. Chem. 1986, 187, 383-400.

[22] V. Kumar, N. P. Suh, Polym. Eng. Sci. 1990, 30, 1323-1329.

[23] C. T. Rueden, J. Schindelin, M. C. Hiner, B. E. DeZonia, A. E. Walter, E. T. Arena, K. W. Eliceiri, BMC Bioinformatics 2017, 18, 529.

[24] MATLAB, Matlab Version 2016a, Tha Mathworks Inc., Natick, Massachusetts, 2016.

[25] L. Chen, M. S. Hoo Fatt, J. Mater. Sci. 2013, 48, 6786-6796.

[26]Y. Shen, Int. J. Fatigue 2001, 23, 491-497.

[27] D. Zenkert, M. Burman, Compos. Sci. Technol. 2009, 69, 785-792. 